\title{
AEBP1 upregulation confers acquired resistance to BRAF (V600E) inhibition in melanoma
}

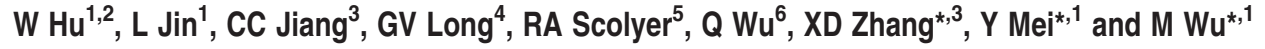

An activating BRAF (V600E) kinase mutation occurs in approximately half of melanomas. Recent clinical studies have demonstrated that vemurafenib (PLX4032) and dabrafenib, potent and selective inhibitors of mutant v-raf murine sarcoma viral oncogene homolog B1 (BRAF), exhibit remarkable activities in patients with V600 BRAF mutant melanomas. However, acquired drug resistance invariably develops after the initial treatment. Identification of acquired resistance mechanisms may inform the development of new therapies that elicit long-term responses of melanomas to BRAF inhibitors. Here we report that increased expression of AEBP1 (adipocyte enhancer-binding protein 1) confers acquired resistance to BRAF inhibition in melanoma. AEBP1 is shown to be highly upregulated in PLX4032-resistant melanoma cells because of the hyperactivation of the PI3K/AktCAMP response element-binding protein (CREB) signaling pathway. This upregulates AEBP1 expression and thus leads to the activation of NF- $\kappa$ B via accelerating $\mid \kappa B$ a degradation. In addition, inhibition of the PI3K/Akt-CREB-AEBP1-NF- $\kappa$ B pathway greatly reverses the PLX4032-resistant phenotype of melanoma cells. Furthermore, increased expression of AEBP1 is validated in post-treatment tumors in patients with acquired resistance to BRAF inhibitor. Therefore, these results reveal a novel PI3K/AktCREB-AEBP1-NF- $\kappa$ B pathway whose activation contributes to acquired resistance to BRAF inhibition, and suggest that this pathway, particularly AEBP1, may represent a novel therapeutic target for treating BRAF inhibitor-resistant melanoma.

Cell Death and Disease (2013) 4, e914; doi:10.1038/cddis.2013.441; published online 7 November 2013

Subject Category: Cancer

Mutant-v-raf murine sarcoma viral oncogene homolog B1 (BRAF) is found in approximately $50 \%$ of melanomas ${ }^{1}$ and induces constituent MEK/extracellular signal regulated kinase (ERK) signaling in melanoma cells. ${ }^{2}$ The most common mutation in BRAF is a substitution of valine with glutamic acid at amino-acid position 600 (V600E) in exon 15..$^{1,3}$ The potent and selective BRAF (V600E) inhibitors, ${ }^{4}$ vemurafenib (PLX 4032) and dabrafenib (GSK2118436), have significantly improved survival for patients with V600 BRAF-mutant metastatic melanoma. ${ }^{5-8}$ Eventually, the majority of patients relapsed with a median progression-free survival period of 57 months, indicating the acquisition of resistance to BRAF inhibition in these patients. ${ }^{5,7,8}$

Over the past 2 years, tremendous efforts have been directed toward understanding the molecular mechanisms of acquired BRAF inhibitor resistance. Emerging evidence suggests that NRAS mutations, amplification of BRAF (V600E) and overexpression of v-raf-leukemia viral oncogene 1 or COT lead to reactivation of MEK that counteracts the inhibition of BRAF. ${ }^{9-12}$ In addition, re-activated IGF-1R/PI3K and FGFR3/RAS/MAPK signaling pathways were shown to have an important role in the development of resistance to BRAF inhibitors. ${ }^{13,14}$ More recently, aberrant expression of splicing isoforms of BRAF (V600E) that dimerize in a RASindependent manner has been shown to mediate acquired BRAF inhibitor resistance. ${ }^{15}$ Upregulated expression of receptor tyrosine kinase and FOXD3 is also associated with BRAF inhibitor resistance. ${ }^{11,16,17}$ Given the diversity and complexity of these identified signaling pathways associated with BRAF inhibitor resistance, fully defining the underlying mechanisms of resistance is still a priority to develop rational strategies to secure long-term responses of mutant BRAF melanomas to specific inhibitors.

To identify novel mechanism(s) of resistance to BRAF inhibition, we generated PLX4032-resistant cell lines by chronic exposure of a BRAF (V600E)-positive melanoma cell line to PLX4032. Here we report AEBP1 as a novel PLX4032 resistance-associated gene. AEBP1 (adipocyte enhancerbinding protein 1) is highly upregulated in PLX4032-resistant melanoma cells because of hyperactivation of the PI3K/AktcAMP response element-binding protein (CREB) signaling pathway. Increased expression of AEBP1 is shown to confer acquired PLX4032 resistance by facilitating activation of $\mathrm{NF}-\kappa \mathrm{B}$. We also show that AEBP1 is indeed upregulated in post-treatment tumors in patients with acquired resistance to BRAF inhibitor. Collectively, these data suggest that the

\footnotetext{
${ }^{1}$ Hefei National Laboratory for Physical Sciences at Microscale and School of Life Sciences, University of Science and Technology of China, Anhui, China; ${ }^{2}$ Department of Immunology, Anhui Medical University, Anhui, China; ${ }^{3}$ School of Biomedical Science and Pharmacy, University of Newcastle, Newcastle, NSW, Australia; ${ }^{4}$ Melanoma Institute Australia and Westmead Institute for Cancer Research, University of Sydney, Sydney, NSW, Australia; ${ }^{5}$ Discipline of Pathology, University of Sydney; Tissue Pathology and Diagnostic Oncology, Royal Prince Alfred Hospital; Melanoma Institute Australia, Sydney, NSW, Australia and ${ }^{6}$ State Key Laboratory of Cellular Stress Biology, School of Life sciences, Xiamen University, Xiamen, China

*Corresponding author: XD Zhang or Y Mei or M Wu, Molecular Cell Biology, School of Life Science, University of Science and Technology of China, Huangshan Road, Hefei 230027, China. Tel: +86 551 3607324; Fax: +86 551 3606264; E-mails: xu.zhang@newcastle.edu.au or meiyide@ustc.edu.cn or wumian@ustc.edu.cn Keywords: AEBP1; BRAF; melanoma

Abbreviations: BRAF, v-raf murine sarcoma viral oncogene homolog B1; AEBP1, adipocyte enhancer-binding protein 1; BR, binding region; PARP, poly (ADP-ribose) polymerase; CREB, cAMP response element-binding protein; ERK, extracellular signal regulated kinase; ChIP, chromatin immunoprecipitation; shRNA, small harpin RNA
}

Received 17.7.13; revised 30.9.13; accepted 04.10.13; Edited by G Raschellá 
PI3K/Akt-CREB-AEBP1-NF- $\kappa$ B pathway has an important role in the development of BRAF inhibitor resistance in mutant BRAF melanomas.

\section{Results}

AEBP1 confers acquired resistance to BRAF (V600E) inhibition in melanoma. To identify novel molecular mechanism(s) of resistance, we generated PLX4032-resistant cell lines by chronic exposure of the BRAF (V600E)positive parental melanoma cell line Mel-CV to PLX4032. After approximately 6 months of continuous selection, 24 independent PLX4032-resistant clones (Mel-CVR1-MelCVR24) were generated. The PLX4032-resistance phenotype of Mel-CVR18 and Mel-CVR21 clones was confirmed by MTT and long-term colony formation assays (Supplementary Figures $1 \mathrm{~A}$ and $\mathrm{B})$. These two clones were then subjected to cDNA microarray analysis to identify potential PLX4032 resistance-associated genes. Through the cDNA microarray analysis, AEBP1 was revealed to be most dramatically upregulated in both Mel-CVR18 and Mel-CVR21 compared with Mel-CV cells (Figure 1a). Real-time RT-PCR analysis showed that most of the PLX4032-resistant Mel-CVR sublines (18 of 24) exhibited a more than twofold increase in AEBP1 mRNA expression compared with the Mel-CV cell line (Figure 1b). The subsequent western blot analysis also confirmed the upregulation of AEBP1 protein expression in PLX4032-resistant Mel-CVR cells (Supplementary Figure 2). As Mel-CVR18 and Mel-CVR21 cells showed similar responses to PLX4032 treatment (Supplementary Figures $1 \mathrm{~A}$ and $\mathrm{B}$ ), in the subsequent functional studies only Mel-CVR18 was used. For convenience, Mel-CVR18 was hereafter referred to as Mel-CVR. To exclude the possibility that AEBP1 upregulation only occurs in Mel-CVR cell type, we also generated other two PLX4032-resistant cell lines from different melanoma cell types Mel-RMu and SK-Mel-28 by chronic exposure to PLX4032. As was expected, both mRNA and protein levels of AEBP1 were upregulated in these PLX4032-resistant cell lines (Supplementary Figure 3).

We next determined whether upregulation of AEBP1 expression confers acquired PLX4032 resistance to melanoma cells. We first knocked down AEBP1 in Mel-CVR cells. Compared with control cells, AEBP1 knockdown Mel-CVR cells showed a dramatic decrease in cell viability in response to PLX4032 treatment (Figure 1c). Accordingly, AEBP1 knockdown Mel-CVR cells exhibited high levels of caspase3 activation and poly(ADP-ribose) polymerase (PARP) cleavage compared with control cells after PLX4032 treatment (Figure 1d). Similar results were obtained when AEBP1 was knocked down in Mel-RMuR and SK-Mel-28R cells (Supplementary Figure 4). We next evaluated the effect of AEBP1 on PLX4032-induced inhibition on cell viability in Mel-CV cells. As shown in Figure 1e, the inhibitory effect of PLX-4032 on cell viability was greatly reversed by ectopic expression of AEBP1 in Mel-CV cells. Correlated with this, AEBP1 overexpression resulted in a great decrease in PLX4032-induced caspase-3 activation and PARP cleavage (Figure 1f). Furthermore, long-term colony formation assay also revealed that when AEBP1 was knocked down in Mel-CVR-, Mel-RMuR- and SK-Mel-28R-resistant cells, these cells became sensitive to PLX4032 treatment (Figure 1g and Supplementary Figure 5). Taken together, these results strongly suggest the important role of AEBP1 in the development of acquired resistance to BRAF inhibition.

AEBP1 is regulated by the PI3K/Akt-CREB pathway. To investigate the molecular mechanism whereby AEBP1 is upregulated in Mel-CVR cells, we first inspected the genomic sequence upstream of AEBP1 coding region by using the JASPAR software. ${ }^{18}$ One putative CREB-binding site located 761-753 bp upstream of AEBP1 translational start site was identified (Figure 2a). To verify this potential CREB-binding site is indeed responsive to CREB, a series of pGL3-based luciferase reporter plasmids containing the wild-type CREBbinding region (BR), a mutant CREB-BR or deletion of CREB-BR were generated (Figure $2 b$ ). These plasmids were individually transfected into Mel-CV and Mel-CVR cells with or without CREB knockdown, followed by the measurement of their transcriptional activities using luciferase assays. As shown in Figure 2c, pGL3 luciferase reporter plasmids containing the wild-type CREB-BR (WT and m4), but not the mutant plasmids (m1, m2, m3 and m5), showed a CREBresponsive transcriptional activity in both Mel-CV and Mel-CVR cells. Of note, the transcriptional activities of pGL3-AEBP-WT and pGL3-AEBP-m4 were much higher in Mel-CVR cells than those in Mel-CV cells (Figure 2c), indicating that the basal CREB transcriptional activity may be higher in Mel-CVR cells than that in Mel-CV cells. Given that the transcriptional activity of CREB is induced by its phosphorylation at $S 133,{ }^{19,20}$ we sought to evaluate the effects of wild-type CREB and its mutant CREB S133A on the AEBP1 promoter activity. As shown in Figure 2d, wildtype CREB, but not CREB S133A, increased the transcriptional activity of the AEBP1 promoter in a dose-dependent manner. The subsequent chromatin immunoprecipitation (ChIP) assays showed that the chromatin fragments, corresponding to the putative CREB BR, were specifically present in anti-CREB immunoprecipitates from both Mel-CV and Mel-CVR cells (Figure 2e), which was, however, diminished by CREB knockdown (Figure 2e). In addition, knockdown of CREB resulted in a dramatic decrease in AEBP1 mRNA levels in Mel-CVR cells (Figure 2f). These results suggest that transcriptional regulation of $A E B P 1$ is mediated by CREB. The importance of the CREB-AEBP1 axis in acquired PLX4032 resistance was also confirmed by the finding that knockdown of either CREB or AEBP1 increased PLX4032-induced inhibition of viability in Mel-CVR cells (Figure $2 \mathrm{~g}$ ).

Intriguingly, in contrast to Mel-CVR cells, CREB knockdown only led to a subtle decrease in AEBP1 mRNA levels in Mel-CV cells (Figure 2f). This discrepancy further suggests that Mel-CVR cells possess higher CREB transcriptional activity than Mel-CV cells. In support of this conclusion, we also found that levels of phosphorylated CREB (S133) were much higher in Mel-CVR cells than those in Mel-CV cells, although total CREB protein showed similar levels in these two cells (Figure 2h). Furthermore, Mel-CVR cells were shown to exhibit higher levels of phosphorylated Akt (S473) (p-Akt) than Mel-CV cells (Figure 2h), indicating that PI3K/Akt signaling is enhanced in PLX4032-resistant melanoma cells. 
a

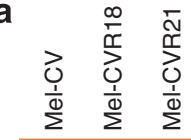

C

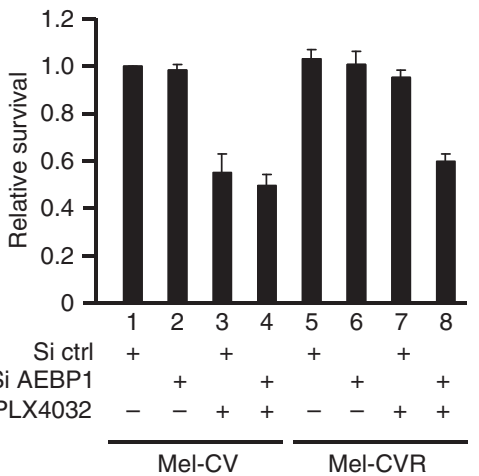

d

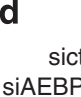

SIAEBP
WASH1 PDPN CXCL PTPRN2 PII RN PDGFRL ENPP1 MED27 ALG10B PDGFRA RFX2 NEK2 XPR1 EGFR ABCD5 AEBP1 ATP8A2 BATF TLK2 EIF2C3 PDGFRB PVT1 NOXA1

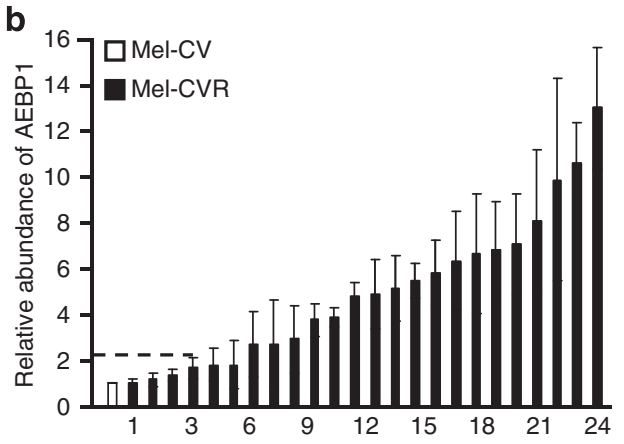

$\begin{array}{lll}-1.8 & 1.8 & 4.0\end{array}$

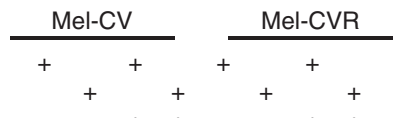

PLX4032 - $-++\ldots++$

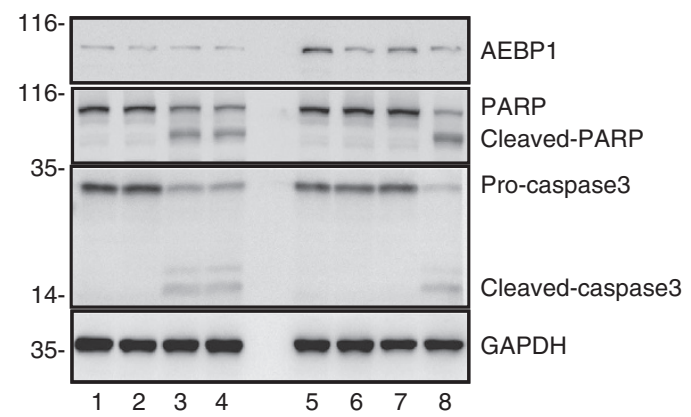

e

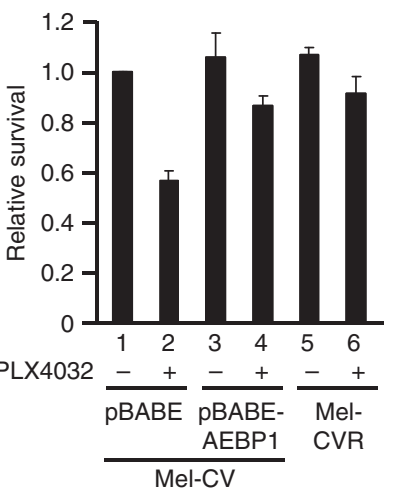

f
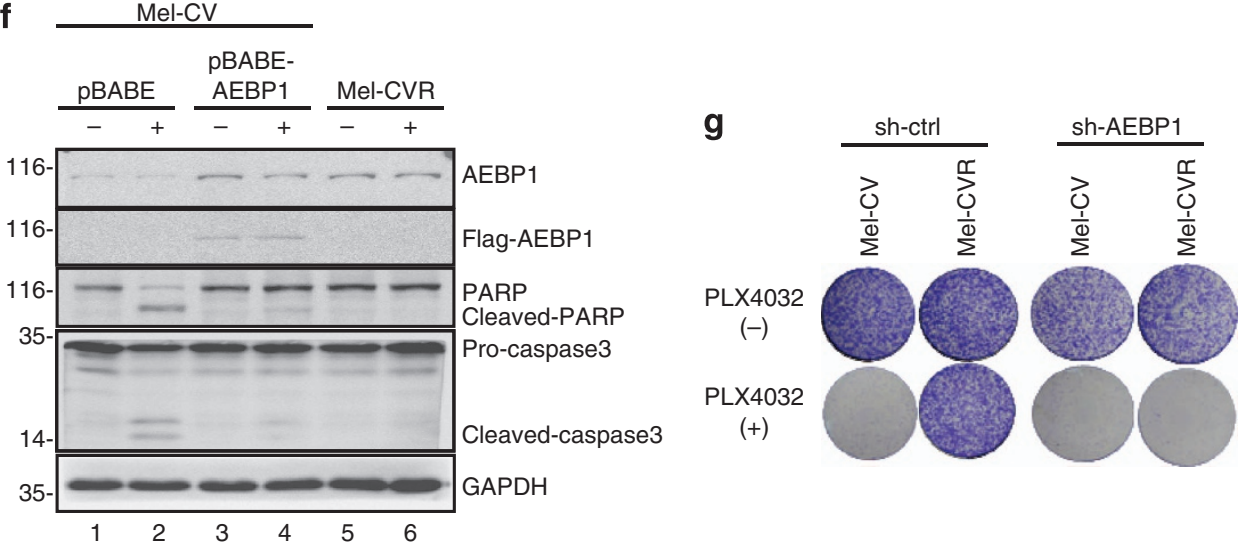

Figure 1 Identification of AEBP1 as a novel PLX4032 resistance-associated gene. (a) Total RNA from Mel-CV parental cell line and two PLX4032-resistant clones (Mel-CVR18 and Mel-CVR21) was individually subjected to cDNA microarray analysis. The filtered microarray data were subjected to unsupervised hierarchical clustering analysis. The metric was set as the Euclidean distance. (b) Real-time RT-PCR analysis of AEBP1 mRNA expression in Mel-CV parental cell line and 24 isolated PLX4032resistant clones (Mel-CVR1-Mel-CVR24). (c) Mel-CV and Mel-CVR cells were individually transfected with either control siRNA or AEBP1 siRNA. $24 \mathrm{~h}$ after transfection, cells were treated with $5 \mu \mathrm{M}$ PLX4032 for an additional $36 \mathrm{~h}$. Viability of cells was determined using MTT assays by measuring the absorbance at $490 \mathrm{~nm}$ in a microplate reader. (d) Mel-CV and Mel-CVR cells were individually transfected with either control siRNA or AEBP1 siRNA. $24 \mathrm{~h}$ after transfection, cells were treated with $5 \mu \mathrm{M}$ PLX4032 or mock control for another $36 \mathrm{~h}$. Cell lysates were then subjected to western blot analysis with the indicated antibodies. (e) Mel-CV cells expressing AEBP1 or control protein and Mel-CVR cells were treated with $5 \mu \mathrm{M}$ PLX4032 or mock control for $36 \mathrm{~h}$. Viability of cells was determined using MTT assays by measuring the absorbance at $490 \mathrm{~nm}$ in a microplate reader. (f) Mel-CV cells expressing AEBP1 or control protein and Mel-CVR cells were treated with $5 \mu \mathrm{M}$ PLX4032 or mock control for $36 \mathrm{~h}$. Cell lysates were analyzed by western blot analysis with the indicated antibodies. (g) Long-term colony formation assay of Mel-CV and Mel-CVR cells with and without stable knockdown of AEBP1. Cells were grown in the absence or presence of $3 \mu \mathrm{M}$ PLX4032 for 3 weeks. All dishes were fixed at the same time, stained and photographed 
a

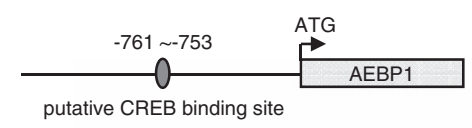

b

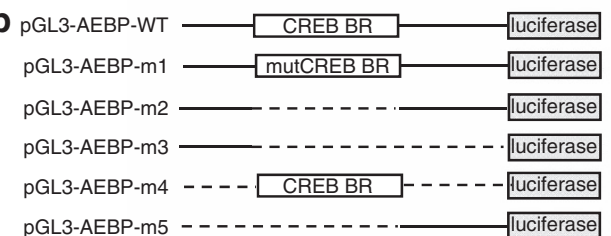

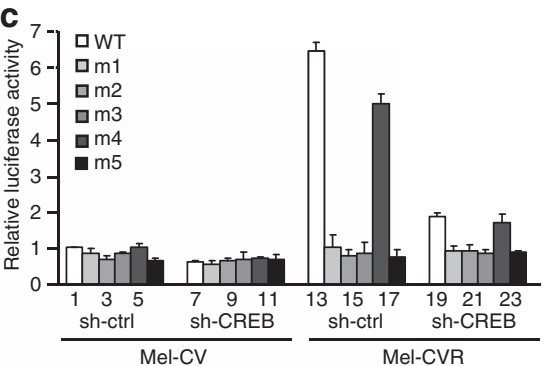

e d

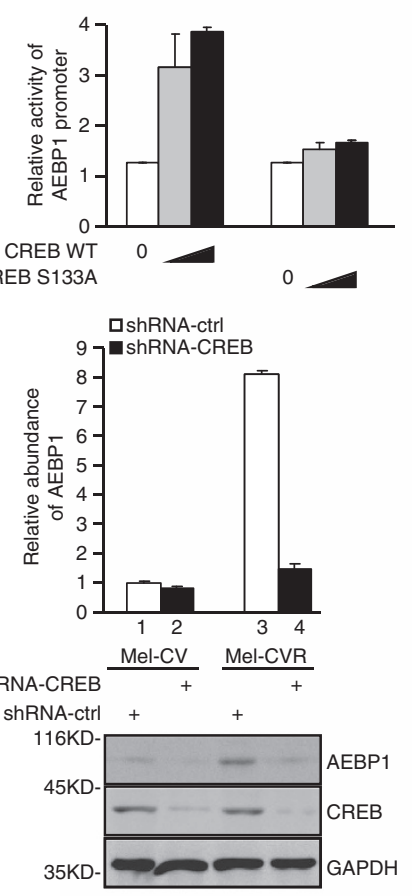

h

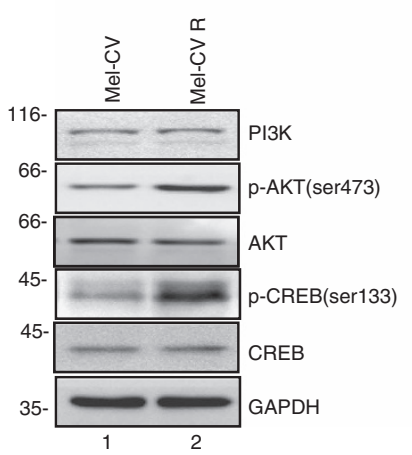

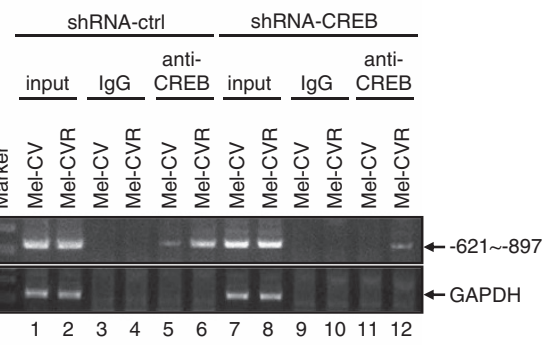

g

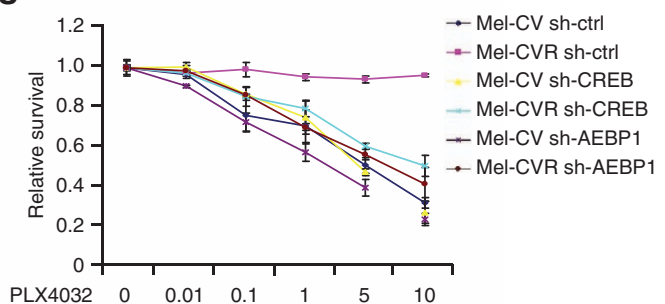

(UM)

i

$\frac{\text { Mel-CV }}{\text { U0126 - + - + }} \frac{\text { Mel-CVR }}{-+--+-}$
Y294002 - + + +

LY294002 - + - + --+--+
PLX4032 - - + + $1---+++$

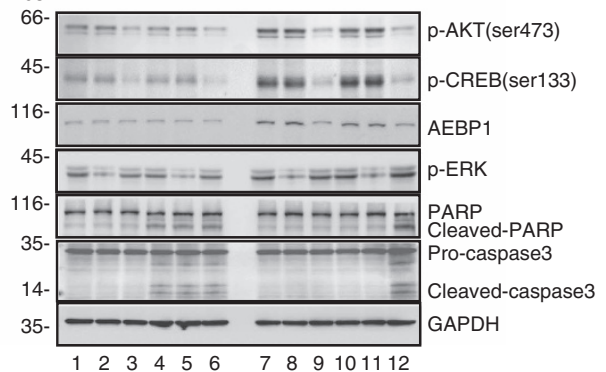

Figure 2 Involvement of PI3K/Akt/CREB in the upregulation of AEBP1. (a) Schematic illustration of the putative CREB-binding region located $761-753 \mathrm{bp}$ upstream of the AEBP1 translational start site. (b) Schematic illustration of pGL3-basic-based reporter constructs used in luciferase assays to examine the transcriptional activity of the CREB-BR. Dotted lines indicated the deleted region. (c) Mel-CV and Mel-CVR cells with and without CREB knocked down by shRNA were co-transfected with the indicated reporter constructs and Renilla luciferase plasmid. $24 \mathrm{~h}$ after transfection, transcriptional activity was determined by luciferase assays. (d) Mel-CV cells were transfected with pGL3-AEBP-WT plus increasing amounts of Flag-CREB or Flag-CREB S133A. $24 \mathrm{~h}$ after transfection, transcriptional activity was determined by luciferase assays. (e) ChIP analysis showed binding of CREB to the CREB-BR. Input and immunoprecipitated DNA from Mel-CV and Mel-CVR cells with and without CREB knocked down by shRNA were amplified by PCR with the primer pair complementary to -897 to -621 of the AEBP1 promoter. An isotype-matched lgG was used as a negative control. (f) Real-time RT-PCR analysis of AEBP1 mRNA expression in Mel-CV and Mel-CVR cells with or without CREB knocked down by shRNA. CREB and AEBP1 levels were evaluated by western blot analysis. (g) Mel-CV and Mel-CVR cells with or without stable knockdown of CREB or AEBP1 were individually treated with indicated concentration of PLX4032 for $36 \mathrm{~h}$. Viability of cells was then examined by MTT assays. (h) Lysates from Mel-CV and Mel-CVR cells were subjected to western blot analysis with the indicated antibodies. (i) Mel-CV and Mel-CVR cells were pre-treated with U0126, LY294002 or DMSO for $4 \mathrm{~h}$, followed by the treatment with $5 \mu$ M PLX4032 for an additional $36 \mathrm{~h}$. Cell lysates were then analyzed by western blot analysis with the indicated antibodies 
The similar results were also obtained in other PLX4032resistant Mel-CVR, Mel-RMuR and SK-Mel-28R clones (Supplementary Figure 6). To confirm this, Mel-CV and Mel-CVR cells were treated with PLX4032 for prolonged periods. The remaining live cells were assayed for Akt activation by evaluating protein levels of $p-A K T$. As shown in Supplementary Figure 7A, PLX4032 treatment resulted in progressive activation of Akt and CREB in Mel-CV cells, which was correlated well with the gradual upregulation of AEBP1 expression by PLX4032 treatment (Supplementary Figure $7 \mathrm{~B}$ ), indicating that activation of Akt is associated with acquired PLX4032 resistance. In contrast to Mel-CV cells, the enhancing effect of PLX4032 on activation of the PI3K/AktCREB pathway and upregulation of AEBP1 expression was minimal in Mel-CVR cells (Supplementary Figures 7A and B). This suggests that the PI3K/Akt-CREB-AEBP1 pathway may have already been fully activated in Mel-CVR cells.

The PI3K/Akt pathway has previously been shown to induce CREB activation by promoting its phosphorylation at S133. ${ }^{19,20}$ To determine the specific role of the PI3K/Akt pathway in mediating CREB activation and AEBP1 upregulation, Mel-CV and Mel-CVR cells were treated with LY294002 or U0126, which inhibits the PI3K/Akt and MEK/ERK pathways, respectively. As shown in Figure 2i, LY294002 treatment, but not U0126 treatment, led to a dramatic decrease in both $\mathrm{p}$-CREB and AEBP1 protein levels in Mel-CVR cells with or without PLX4032 treatment. More importantly, pre-treatment of Mel-CVR with LY294002 led to a strong increase in PARP cleavage and caspase-3 activation (Figure 2i) and a dramatic decrease in cell viability in response to PLX4032 treatment (Supplementary Figure 8). Similar results were also obtained from PLX4032-resistant MelRMuR and SK-Mel-28R cells that were pre-treated with LY294002 (Supplementary Figure 9). However, when expression levels of $p$-CREB and AEBP were induced by ectopic expression of constitutively active form of AKT (Myr-AKT) in Mel-CV and Mel-RMu cells (Supplementary Figure 10A), these cells became resistant to PLX4032 treatment (Supplementary Figures $10 \mathrm{~B}$ and $\mathrm{C}$ ). We also found that the LY294002 pretreatment did not further enhance PLX4032mediated cell viability inhibition in AEBP1 knockdown Mel-CVR cells (Supplementary Figure 8), indicating that AEBP1 functions in full alignment with PI3K/Akt activity in the development of acquired resistance to PLX4032. Taken together, these data suggest that AEBP1 is upregulated by the PI3K/Akt-CREB pathway, through which AEBP1 contributes to the acquired resistance of mutant BRAF melanoma cells to BRAF inhibition.

\footnotetext{
AEBP1 mediates activation of NF- $\kappa \mathrm{B}$ and thereby contributes to acquired resistance to BRAF inhibition. We next investigated how AEBP1 contributes to acquired resistance to BRAF inhibition. AEBP1 has recently been recognized as a positive regulator of $\mathrm{NF}-\kappa \mathrm{B}$ activation through accelerating degradation of $\mathrm{I} \kappa \mathrm{B} \alpha{ }^{21}$ It has also been shown that NF- $\kappa \mathrm{B}$ activation protects cells from BRAF inhibition-induced cell death. ${ }^{22}$ We therefore asked whether AEBP1 could activate NF- $\kappa \mathrm{B}$ and thus confer resistance to BRAF inhibition. Consistent with the previous report, with the increasing expression of AEBP1, levels of $\left.\right|_{\kappa} \mathrm{B} \alpha$ were
}

concomitantly decreased in Mel-CV cells (Figure 3a), thereby leading to the gradual activation of $\mathrm{NF}-\kappa \mathrm{B}$ (Figure $3 \mathrm{~b}$ ). Similar to AEBP1, CREB also showed a similar decrease in $\mathrm{I}_{\kappa} \mathrm{B} \alpha$ protein levels and an increase in NF- $\kappa$ B activation (Figure $3 a$, lanes 4-6 and Figure 3b). This effect of CREB appeared to require its transcriptional activity, as revealed by the finding that wild-type CREB, but not CREB S133A, promoted NF- $\kappa \mathrm{B}$ activation (Figures $3 c$ and $d$ ). The failure of $C R E B$ to induce $\mathrm{NF}-\kappa \mathrm{B}$ activation in AEBP1 knockdown Mel-CV cells (Figure 3e) suggests that AEBP1 is the downstream mediator of CREB to promote NF- $\kappa$ B activation.

Given the upregulation of AEBP1 expression in Mel-CVR, Mel-RMuR and SK-Mel-28R cells, we sought to determine whether this resulted in NF- $\kappa$ B activation in these cells. As shown in Figure $3 f$ and Supplementary Figure 11A, Mel-CVR, Mel-RMuR and SK-Mel-28R cells indeed showed higher NF- $\kappa \mathrm{B}$ activity and lower $\mathrm{I}_{\kappa} \mathrm{B} \alpha$ expression compared with their corresponding sensitive parental cells. Correlated with the lower levels of $\mathrm{I}_{\kappa} \mathrm{B} \alpha$ in Mel-CVR cells, the half-life of $\mathrm{I}_{\kappa} \mathrm{B} \alpha$ in MelCVR cells was much shorter than that in Mel-CV cells (Figure 3g). However, when AEBP1 was knocked down in Mel-CVR cells, the half-life of $I_{\kappa} \mathrm{B} \alpha$ was significantly prolonged (Figure $3 g$ ). In addition, knockdown of either AEBP1 or CREB showed strong inhibitory effect on the NF- $\kappa$ B transcriptional activity in Mel-CVR, Mel-RMuR and SK-Mel-28R cells (Figure $3 \mathrm{~h}$ and Supplementary Figure 11B). Treatment with LY294002, but not U0126, greatly inhibited the NF- $\kappa \mathrm{B}$ transcriptional activity in Mel-CVR, Mel-RMuR and SK-Mel28R cells (Figure $3 i$ and Supplementary Figure 11C). Induction of constitutively active form of AKT (Myr-AKT) in Mel-CV and Mel-RMu cells strongly enhanced NF- $\kappa \mathrm{B}$ activity (Supplementary Figure 11D). These results indicate that the hyperactivated PI3K/Akt-CREB-AEBP1 pathway is responsible for the increased NF- $\kappa$ B activity in PLX4032-resistant melanoma cells.

To further determine the physiological significance of AEBP1-mediated NF- $\kappa$ B activation in relation to the acquired resistance to PLX4032, we used PDTC, BAY-11-7082 and ALLN, which were specific inhibitors of NF- $\kappa$ B activation. When NF- $\kappa$ B activation was inhibited by PDTC, BAY-11-7082 or ALLN, Mel-CVR cells exhibited a dramatic decrease in cell viability (Figure 3j and Supplementary Figure 12A) and a strong increase in PARP cleavage and caspase-3 activation in response to $\mathrm{PLX} 4032$ treatment (Figure $3 \mathrm{k}$ and Supplementary Figure 12B). Similarly, when NF- $\kappa$ B activation was inhibited by ectopic expression of a non-degradable mutant of $I_{\kappa} \mathrm{B} \alpha\left(\mathrm{I}_{\kappa} \mathrm{B} \alpha \mathrm{S} 32 \mathrm{AS} 36 \mathrm{~A}\right)$ in Mel-CVR cells, the cells became sensitive to PLX4032 treatment (Supplementary Figures $13 \mathrm{~A}$ and $\mathrm{B})$. Conversely, when NF- $\kappa \mathrm{B}$ activity was induced by $\mathrm{I}_{\kappa} \mathrm{B}$ knockdown in Mel-CV and Mel-RMu cells (Supplementary Figure 13C), these cells became resistant to PLX4032 treatment without causing any change in AEBP1 expression (Supplementary Figures 13D-F). Taken together, these results suggest that $\mathrm{NF}-\kappa \mathrm{B}$ is activated by the $\mathrm{PI}$ KK/AktCREB-AEBP1 pathway, and thus confers BRAF inhibitor resistance to melanoma cells.

Biological significance of increased expression of AEBP1 in acquired resistance to BRAF inhibition. To validate the functional significance of AEBP1 upregulation in 
a

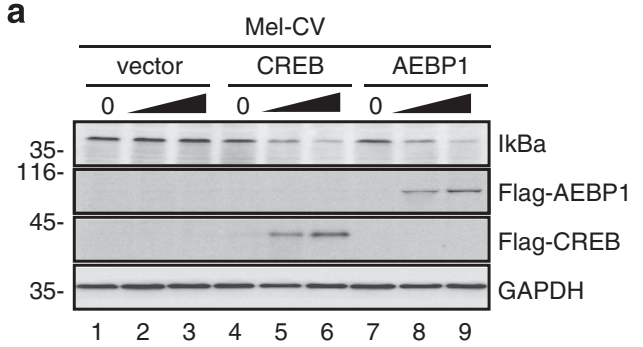

b

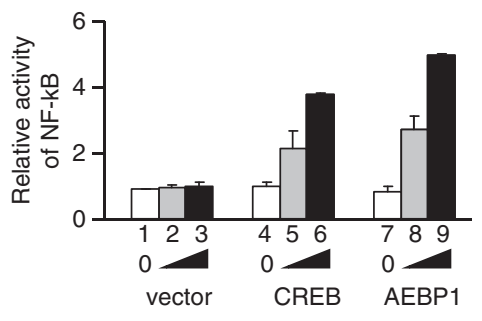

C

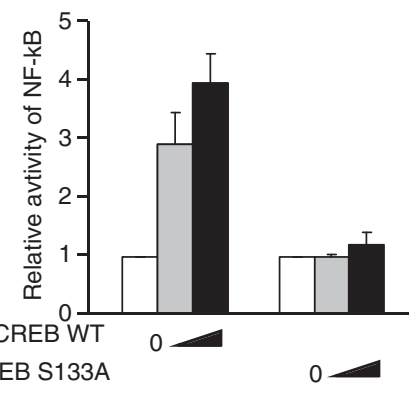

d

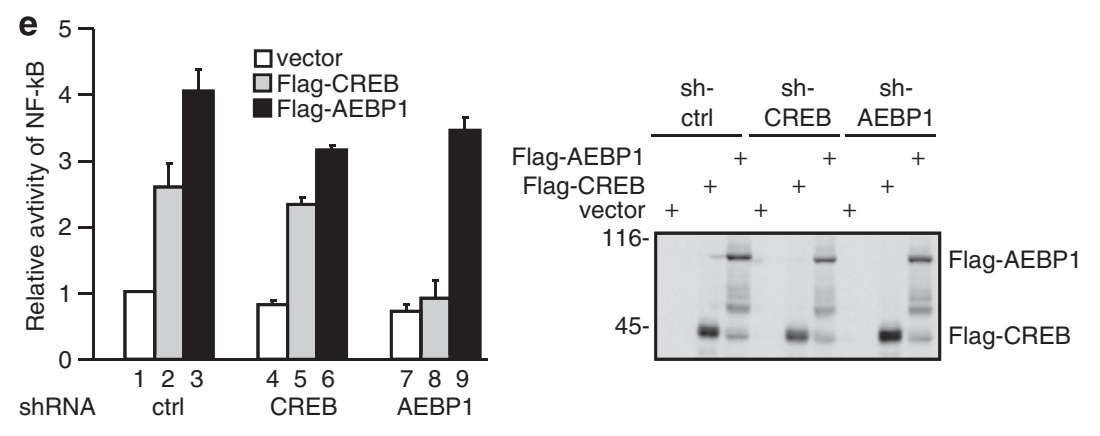

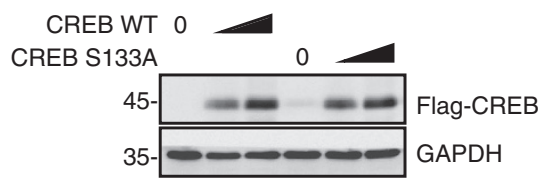

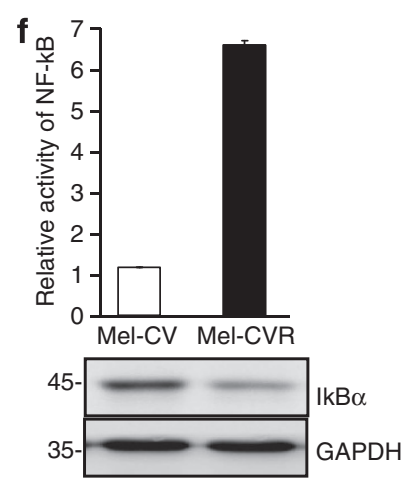

g Mel-CVR

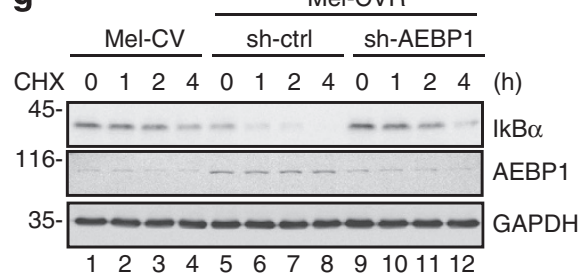

h
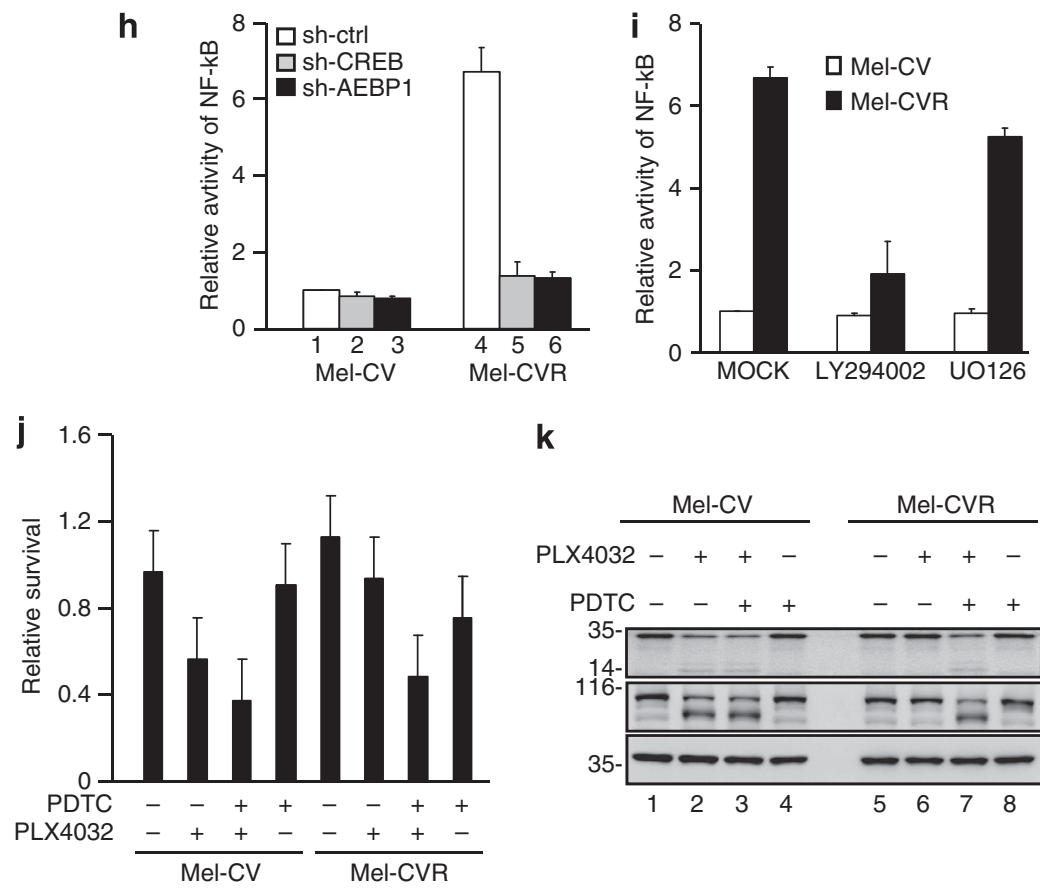

k
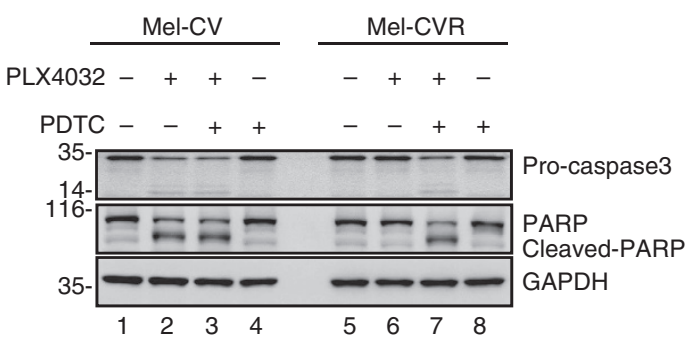
acquired BRAF inhibitor resistance in vivo, we used a xenograft mouse model. Mel-CV and Mel-CVR cells with and without stable knockdown of either CREB or AEBP1 were individually injected subcutaneously into the right flank of nude mice. As shown in Figure $4 \mathrm{a}$, all these melanoma cells developed into tumors of comparable volumes at 15 days after injection, indicating that both CREB and AEBP1 have no effects on melanomagenesis in this xenograft model. After tumor establishment (about $50 \mathrm{~mm}^{3}$ ), mice were treated with PLX4032 $(50 \mathrm{mg} / \mathrm{kg})$ through intraperitoneal (i.p.) injection. After 25 days of the commencement of treatment, the excised tumors were weighed and analyzed for expression of AEBP1 and CREB and activation of caspase-3/7. As shown in Figures $4 a$ and $b$, Mel-CV xenografts were largely abolished by treatment of PLX4032, whereas Mel-CVR xenografts showed little, if any, responses to PLX4032 treatment. However, when AEBP1 or CREB was knocked down in Mel-CVR cells, the xenografts showed the similar shrinkage rates as Mel-CV tumors in response to PLX4032 (Figures $4 a$ and b). This was also confirmed by a significant decrease in xenograft weights (Figure 4c). The knockdown efficiency of AEBP1 or CREB was confirmed by western blot analysis of xenograft samples post treatment with PLX4032 (Figure 4d). Also, knockdown of either AEBP1 or CREB resulted in a dramatic increase in caspase-3/7 activity in Mel-CVR xenografts in response to treatment with PLX4032 (Figure 4e). These results point to an important role of the CREB-AEBP1 axis in antagonizing PLX4032-mediated inhibition of melanoma growth in vivo.

To test whether AEBP1 is involved in development of acquired resistance to BRAF inhibition in melanoma patients, we first took advantage of primary melanoma cell cultures of paired BRAF (V600E) melanoma biopsy samples from four patients pre- and progressed post-treatment with PLX4032. ${ }^{23}$ These melanomas responded initially to PLX4032 but relapsed after various progression-free durations, ${ }^{23}$ indicative of development of acquired resistance. We found that two of four post-treatment cultures expressed lower levels of $I_{\kappa} \mathrm{B} \alpha$ and higher levels of AEBP1 and p-Akt than corresponding pretreatment cells (Figure 4f), consistent with the findings in melanoma cell lines that increased AEBP1 expression, Akt phosphorylation and NF- $\kappa$ B activation were associated with resistance to PLX4032. Similarly, examination of paired BRAF (V600E) melanoma biopsy samples taken from five patients pre- and progressed post-treatment with the BRAF inhibitor dabrafenib by immunohistochemistry revealed that two of five melanomas progression samples displayed increased expression of AEBP1 in comparison with the corresponding samples before treatment (Supplementary Figure 14). Together, these findings suggest that the development of BRAF inhibitor resistance in a sub-group of BRAF (V600E) melanomas is closely associated with upregulation of AEBP1.

\section{Discussion}

Approximately $80 \%$ of patients with mutant BRAF melanomas respond initially to vemurafenib and dabrafenib treatment, but acquired resistance develops in the majority of patients commonly within 1 year. $^{5-8}$ In this study, we have provided evidence that increased expression of AEBP1 contributes to the development of acquired resistance of mutant BRAF melanomas to specific inhibitors.

AEBP1 was originally identified as a transcriptional repressor with both carboxypeptidase and DNA-binding activities, ${ }^{24,25}$ and has been implicated in the regulation of various biological processes. $^{26-28}$ Our data showing that AEBP1 is upregulated in at least a proportion of BRAF (V600E) melanomas that had acquired resistance to PLX4032, and that inhibition of AEBP1 restores its sensitivity to $\mathrm{PLX} 4032$ in resistant cells uncover a previously unidentified function of AEBP1 in association with BRAF inhibitor resistance.

The increase of AEBP1 in BRAF (V600E) melanoma cells with PLX4032 resistance appears to be due to transcriptional upregulation mediated by the transcription factor CREB. It has been well known that the transcriptional activity of CREB is activated via its phosporylation at $\mathrm{S} 133$ by various signaling pathways, such as Akt, MAPK and ERK. ${ }^{19,20,29,30}$ Our finding that Akt inhibition, but not ERK inhibition, results in marked decreases in protein levels of both phosphorylated CREB and AEBP1, pointing to a specific role of PI3K/Akt signaling in activating $C R E B$ and subsequent upregulation of AEBP1 in the context of PLX4032 resistance. Along with upregulation of AEBP1, PI3K/Akt-CREB signaling is also highly activated in PLX4032-resistant melanoma cells, the detailed mechanism underlying this phenomenon still remains to be further determined. Although we did not find the mutations in PTEN or PI3K in Mel-CVR cells (data not shown), it is still possible that the hyper-activation of the PI3K/Akt-CREB pathway in PLX4032-resistant melanoma cells is due to the

Figure 3 Activation of NF- $\kappa$ B by AEBP1. (a) Mel-CV cells were transfected with increasing amounts of Flag-CREB or Flag-AEBP1. 24 h after transfection, cell lysates were analyzed by western blot analysis. (b) Mel-CV cells were co-transfected with pGL3 $\times$ NF- $\kappa$ B-BR construct and increasing amounts of Flag-CREB or Flag-AEBP1. $24 \mathrm{~h}$ after transfection, cell lysates were analyzed by luciferase assays. (c and d) Mel-CV cells were co-transfected with pGL3-3 $\times$ NF- $\kappa$ B-BR construct and increasing amounts of Flag-CREB or Flag-CREB S133A. $24 \mathrm{~h}$ after transfection, cell lysates were subjected to lucifease assays (c) and western blot analysis (d), respectively. (e) Mel-CV cells with and without stable knockdown of CREB or AEBP1 were individually co-transfected with $p G L 3-3 \times$ NF- $k$ B-BR and either Flag-CREB or Flag-AEBP1. $24 \mathrm{~h}$ after transfection, cell lysates were subjected to lucifease assays and western blot analysis, respectively. (f) Mel-CV and Mel-CVR cells were individually transfected with $p G L 3-3 \times N F-\kappa B-B R$ construct together with Renilla luciferase plasmid. $24 \mathrm{~h}$ after transfection, the activity of $\mathrm{NF}-\kappa \mathrm{B}$ was determined by luciferase assays. The expression levels of $I_{\kappa} \mathrm{B} \alpha \mathrm{in} \mathrm{Mel}-\mathrm{CV}$ and Mel-CVR cells were compared by western blot analysis. (g) Mel-CVR cells with or without AEBP1 knocked down by shRNA together with Mel-CV cells were treated with cycloheximide (CHX) for the indicated periods of time. Cell lysates were then analyzed by western blot analysis with the indicated antibodies. (h) Mel-CV and Mel-CVR cells individually expressing control shRNA, CREB shRNA or AEBP1 shRNA were transfected with $p G L 3-3 \times N F-k B-B R$ construct together with Renilla luciferase plasmid. $24 \mathrm{~h}$ after transfection, the activity of NF- $\kappa$ B was determined by luciferase assays. (i) Mel-CV and Mel-CVR cells were co-transfected with pGL3-3 $\times$ NF- $\kappa$ B-BR construct and Renilla luciferase plasmid. $24 \mathrm{~h}$ after trasfection, cells were treated with Ly294002, U0126 or DMSO for another $4 \mathrm{~h}$. The activity of NF- $k \mathrm{~B}$ was then analyzed by luciferase assays. (j) Mel-CV and Mel-CVR cells were pre-treated with or without $1 \mu \mathrm{M}$ PDTC for $2 \mathrm{~h}$, followed by the treatment with $5 \mu \mathrm{M}$ PLX4032 for another $36 \mathrm{~h}$. Cell viability was determined by MTT assays. (k) Mel-CV and Mel-CVR cells were pre-treated with or without $1 \mu \mathrm{M}$ PDTC for $2 \mathrm{~h}$, followed by the treatment with $5 \mu \mathrm{M}$ PLX4032 for an additional $36 \mathrm{~h}$. Cell lysates were then analyzed by western blot analysis 
a

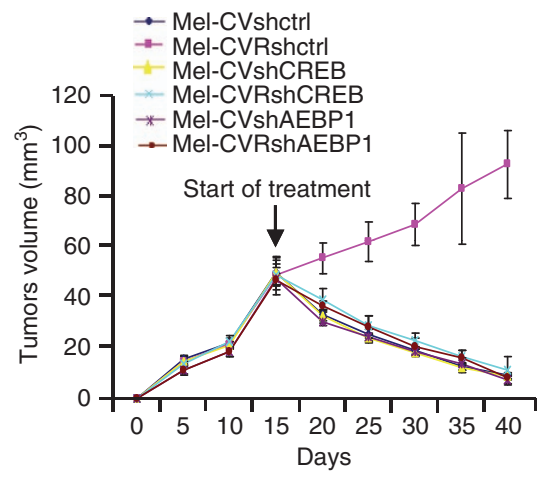

C

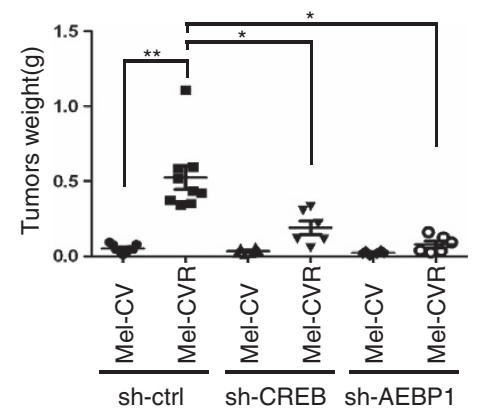

d $\frac{\text { shRNA-ctrl }}{\text { CV CVR }} \frac{\text { shRNA-CREB }}{\text { CV CVR }} \frac{\text { shRNA-AEBP1 }}{\text { CV CVR }}$

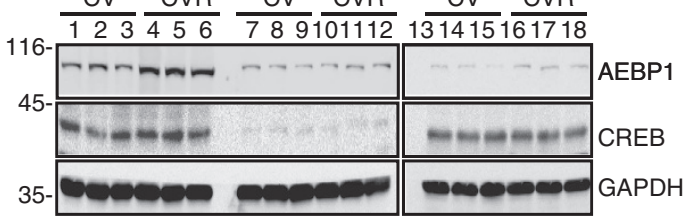

e

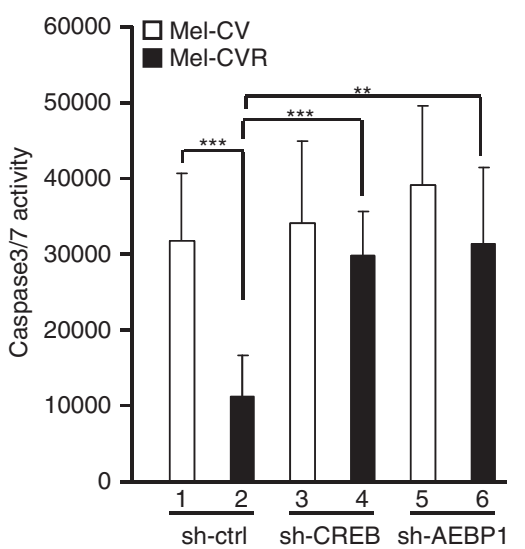

b

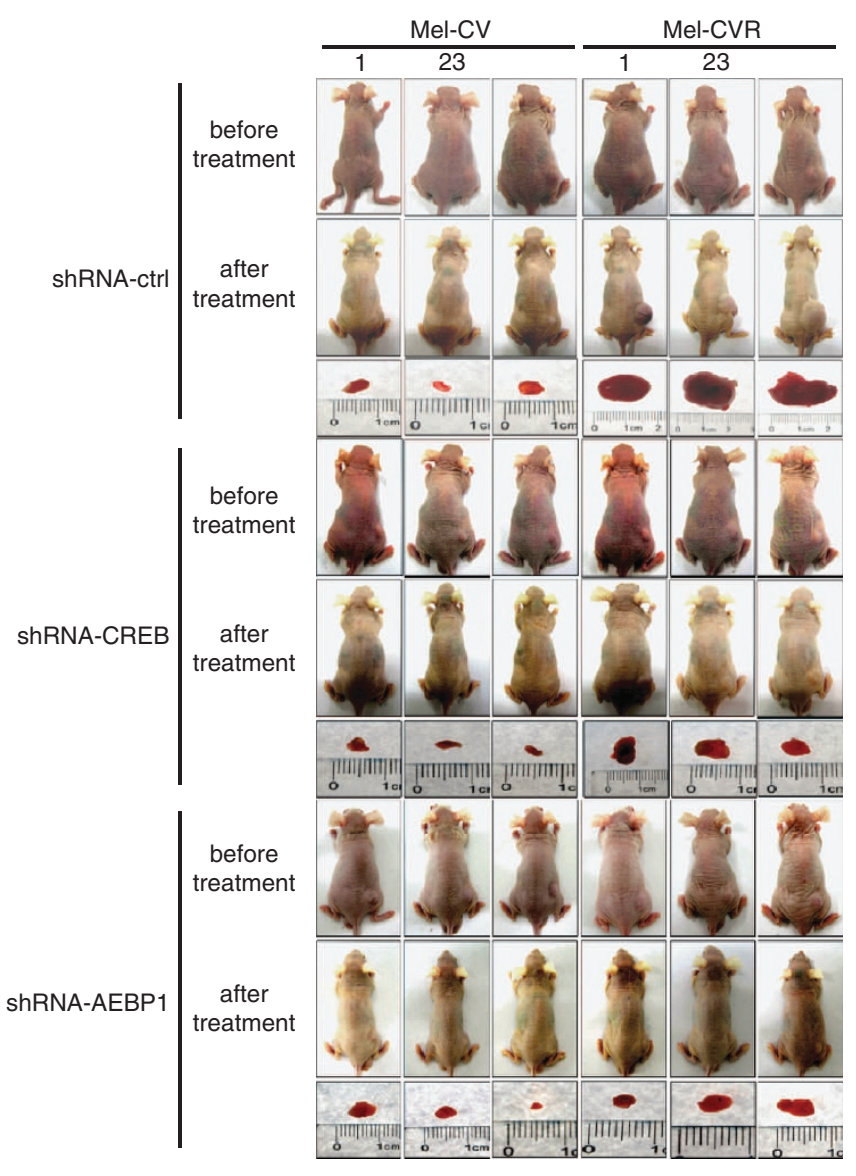

f

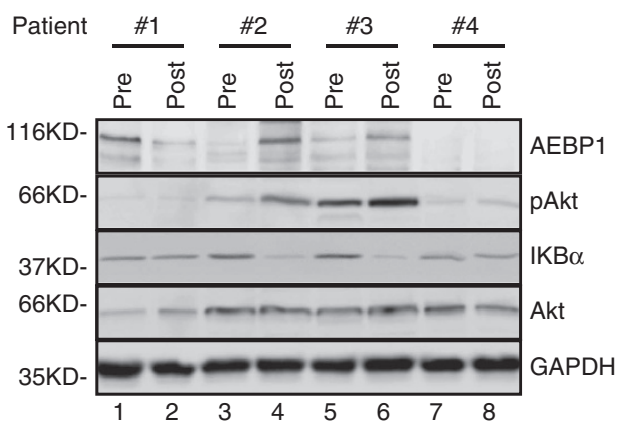

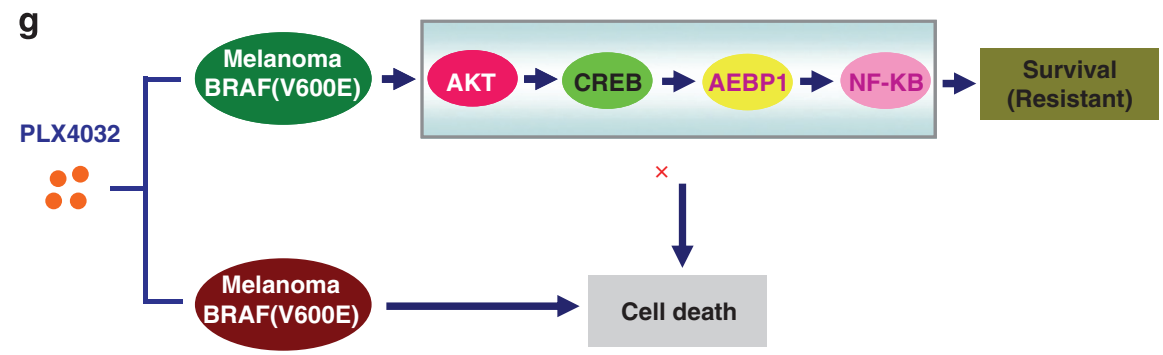


activation of upstream signaling cascade, such as HGF-MET pathway. ${ }^{31,32}$ In this study, we also found that inhibition of the PI3K/Akt-CREB pathway, but not of the MEK/ERK pathway, leads to a dramatic increase in cell death of PLX4032resistant cells. These results indicate that AEBP1 is specifically upregulated by PI3K/Akt-CREB signaling and contributes to the acquired resistance to BRAF inhibition.

In agreement with our data, recent studies have also suggested that increased activation of PI3K/Akt signaling has an important role in the development of BRAF inhibitors resistance. ${ }^{13,33,34}$ AEBP1 has been reported to interact with PTEN and promote its degradation. ${ }^{35}$ Consistently, AEBP1deficient mice exhibit increased PTEN levels and suppressed Akt activation. ${ }^{35}$ Therefore, it is conceivable that AEBP1 may be part of a positive autoregulatory feedback loop that reinforces the PI3K/Akt-CREB signaling axis in BRAF inhibitor-resistant melanoma cells.

Numerous studies have suggested the existence of a crosstalk between $\mathrm{PI} 3 \mathrm{~K} / \mathrm{Akt}$ and $\mathrm{NF}-\kappa \mathrm{B}$ signaling pathways. $\mathrm{PI} 3 \mathrm{~K} / \mathrm{Akt}$ signaling can activate $\mathrm{NF}-\kappa \mathrm{B}$ in response to a variety of stimuli by phosphorylating $\mathrm{I}_{\kappa} \mathrm{K} \alpha\left(\mathrm{I}_{\kappa} \mathrm{B}\right.$ kinase $\left.\alpha\right) .{ }^{36}$ In addition, Akt also phosphorylates the NF- $\kappa \mathrm{B}$ subunit $\mathrm{p} 65$ and increase binding of the NF- $\kappa$ B complex to DNA. ${ }^{37}$ Our results showing that PI3K/Akt-CREB-mediated upregulation of AEBP1 leads to $\mathrm{NF}-\kappa \mathrm{B}$ activation through accelerating $\mathrm{I}_{\kappa} \mathrm{B} \alpha$ degradation in BRAF inhibitor-resistant melanoma cells, together with the finding from another study, ${ }^{21}$ demonstrate that AEBP1 has an important role in the activation of NF- $\kappa$ B. Given that AEBP1 is subjected to transcriptional regulation by $\mathrm{PI} 3 \mathrm{~K} / \mathrm{Akt}$ pathway, these data identify AEBP1 as a novel critical node that connects the PI3K/Akt and NF- $\kappa \mathrm{B}$ signaling pathways.

Although activation of $\mathrm{NF}-\kappa \mathrm{B}$ is critical for melanoma cell growth, ${ }^{38,39}$ whether it is associated with acquired BRAF inhibitors resistance remains uncertain. Our findings that $\mathrm{NF}-\kappa \mathrm{B}$ is highly activated because of the AEBP1 upregulation in PLX4032-resistant melanoma cells, and that inhibition of $\mathrm{NF}-\kappa \mathrm{B}$ reverses the resistance phenotype of the cells, suggest that $\mathrm{NF}-\kappa \mathrm{B}$ activation has an important role in the acquisition of BRAF inhibitor resistance in melanoma cells.

The increased expression of AEBP1 in relapsed melanomas from patients post treatment with BRAF inhibitor suggests that upregulation of AEBP1 is, at least in a proportion of mutant BRAF melanomas, associated with resistance to BRAF inhibition, and provides novel insights into potential therapeutic strategies for the treatment of mutant BRAF melanoma cells that have developed resistance to the inhibitors. In support, an in vivo xenograft mouse model shows that inhibition of AEBP1 greatly enhances anti-tumor effect of PLX4032 on BRAF inhibitor-resistant melanomas.
As mentioned earlier, several mechanisms of BRAF inhibitor resistance have been proposed. In this study, we also examined whether Mel-CVR-, SK-Mel-28R- and Mel-RMuR-resistant cells harbor mutations in NRAS and MEK, and we failed to detect any mutations in either NRAS or MEK in these three resistant cell lines (Supplementary Figure 15). In addition, the previously reported BRAF splice variants were not detected in Mel-CVR cells either (Supplementary Figure 16). These data indicate that activation of AEBP1 represents a unique mechanism of acquired resistance to BRAF inhibition. In summary, this study presents a novel PI3K/Akt-CREB-AEBP1-NF- $\kappa$ B signaling pathway whose activation contributes to acquired resistance to PLX4032 in BRAF-mutant melanoma cells, and identifies AEBP1 as a potential therapeutic target for the treatment of BRAF inhibitor-resistant melanomas (Figure 4g).

\section{Materials and Methods}

Cell culture and reagents. Human melanoma cell lines described previously were cultured in DMEM containing $5 \% \mathrm{FCS}^{40} \mathrm{Mel}-\mathrm{CV}$ and $\mathrm{Mel}-\mathrm{RMu}$ were from lymph node metastases, and SK-Mel-28 was from a primary melanoma. ${ }^{40}$ Primary melanoma cultures of paired BRAF (V600E) melanoma biopsy samples from patients pre- and post-treatment with vemurafenib were established and described previously. ${ }^{22}$ PLX4032 was purchased from Selleck Inc. (Houston, TX, USA). It was dissolved in DMSO to make up stock solutions of $10 \mu \mathrm{M}$. The PI3K inhibitor LY294002 and the MEK inhibitor U0126 were obtained from Sigma (St. Louis, MO, USA). The following antibodies were used in this study: antibodies against AKT (Cell Signaling, Danvers, MA, USA; 2920S), I $\kappa$ B $\alpha$ (Cell Signaling, 4812S), phospho-ERK (Cell Signaling, 4370S), CREB (Cell Signaling, 9197S) and phospho-CREB (Ser133) (Cell Signaling, 4276S); antibodies against AEBP1 (Santa Cruz Biotechnology, Dallas, TX, USA; SC-271374), GAPDH (Santa Cruz Biotechnology, SC-32233), PARP (Santa Cruz Biotechnology, SC-8007); anticaspase-3 (Stressgene, CA-Brockville, ON, Canada; AAP-113) and anti-phospho-AKT (Ser473) (Epitomics, Burlingame, CA, USA; 2118-1). Primary melanoma cell cultures were established as described previously. ${ }^{23}$

Generation of PLX4032-resistant melanoma cell line. Mel-CV cells were initially treated with $20 \mu \mathrm{M}$ PLX4032 for 4 days. The remaining live cells were switched into fresh medium containing $5 \mu \mathrm{M}$ PLX4032 and cultured for about 2 weeks till the colonies became visible. Twenty-four independent resistant colonies (Mel-CVR1-24) were picked and expanded in the culture medium containing $5 \mu \mathrm{M}$ PLX4032. Then the cells were passaged and cultured in the medium containing $5 \mu \mathrm{M}$ PLX4032 for at least 20 weeks before they were used for the subsequent studies.

The similar procedures were used to generate other two PLX4032-resistant cell lines Mel-RMuR and SK-Mel-28R.

Retroviral overexpression of AEBP1. To produce retrovirus expressing AEBP1, HEK293T cells were co-transfected with pBABE-Flag-AEBP1 and $\mathrm{pCL}-$ Ampho packaging vector. $24 \mathrm{~h}$ after transfection, cells were cultured with fresh medium for an additional $24 \mathrm{~h}$. The culture medium containing the retrovirus particles was then centrifuged at $12000 \times g$ for $5 \mathrm{~min}$, and virus in the supernatant was used for melanoma cells infection.

Figure 4 Biological significance of AEBP1 in acquired resistance to PLX4032. (a-e) Mel-CV and Mel-CVR cells with and without stable knockdown of CREB or AEBP1 were individually injected subcutaneously into the right flank of nude mice $(n=6)$. After tumor establishment (about $\left.50 \mathrm{~mm}^{3}\right)$, mice were treated with PLX4032 $(50 \mathrm{mg} / \mathrm{kg}$ ) through intraperitoneal injection for 25 days. (a) Growth curves of xenograft tumors of the indicated cell lines. (b) Representative pictures of tumor-bearing nude mice with pre-treatment and 25 days post treatment of PLX4032. Pictures of tumors excised from the indicated mice 25 days after PLX4032 treatment were also shown. (c) Weights of tumors excised from the indicated mice 25 days after PLX4032 treatment. (d) Lysates from tumors excised from the indicated mice 25 days after PLX4032 treatment were analyzed for AEBP1 and CREB expression by western blot analysis with the indicated antibodies. (e) Lysates from tumors excised from the indicated mice 25 days after PLX4032 treatment were assayed for caspase3/7 activity. (f) Fresh melanoma isolates of paired sets of pre-treatment (pre) and post-relapsed (post) patient specimens from four patients with relapsed $\mathrm{BRAF}^{\mathrm{V} 600 \mathrm{E}}$-expressing melanomas were analyzed for expression of $\mathrm{AEBP} 1$, phospho-Akt and I $\kappa \mathrm{B} \alpha$ by western blot analysis. (g) A schematic illustration of the putative model depicting the important role of AKT-CREB-AEBP1-NF- $\kappa B$ pathway in acquired resistance to PLX4032. ${ }^{*} P<0.05 ;{ }^{* *} P<0.01 ;{ }^{* *} P<0.001$ 
Lentiviral small harpin RNA (shRNA)-mediated knockdown of CREB and AEBP1. To generate lentiviruses expressing CREB, AEBP1 or control shRNAs, HEK293T cells grown on a 6-cm dish were transfected with $2 \mu \mathrm{g}$ of CREB shRNA, AEBP1 shRNA (cloned in PLKO.1) or control vector, $2 \mu \mathrm{g}$ of pREV, $2 \mu \mathrm{g}$ of pGag/Pol/PRE and $1 \mu \mathrm{g}$ of pVSVG. $24 \mathrm{~h}$ after transfection, cells were cultured with DMEM medium containing $20 \%$ FBS for another $24 \mathrm{~h}$. The culture medium containing lentivirus particles was cleaned by centrifugation to get rid of the cell debris at $12000 \times g$ for $5 \mathrm{~min}$, and used for the target cells infection. The shRNA sequences targeting CREB and AEBP were $5^{\prime}$-TACAGCTGGCTAA CAATGG- $3^{\prime}$ and $5^{\prime}$-CGATGACATGGACTATTAC- $3^{\prime}$, respectively.

Dual-luciferase reporter assay. Mel-CV cells were transiently transfected with the indicated luciferase reporter plasmid, together with either Flag-CREB or control vector. Renilla plasmid was also included in each transfection to normalize the transfection efficiency. Firefly and Renilla luciferase activities were analyzed by Dual-Luciferase Reporter Assay system according to the manufacturer's instructions (Promega, Madison, WI, USA). The relative luciferase activities were calculated by normalizing the firely luciferase activity to Renilla luciferase activity. The represented data were mean \pm S.D. of three independent experiments.

Real-time RT-PCR. Total RNA was isolated using Trizol (Sangon Company, Shanghai, China). $1 \mu \mathrm{g}$ of RNA was used to synthesize cDNA using PrimeScriptTM RT reagent kit (TaKaRa, Otsu, Shiga, Japan; DRR037A) according to the manufacturer's instruction. Real-time PCR was performed using SYBR premix EX Taq (TaKaRa) and ROX and analyzed with Stratagene Mx3000p (Agilent Technologies, Santa Clara, CA, USA). Real-time primer sequences for AEBP1 were as follows: $5^{\prime}$ - AGGGCAGA GACTCCAGCAT $-3^{\prime}$ and $5^{\prime}$ - AGAGCACCCCAGCACCTC $-3^{\prime}$.

Colony formation assay. Mel-CV and Mel-CVR cells with and without stable knockdown of CREB or AEBP1 were plated at a density of 20000 cells per well on a six-well plate in the normal medium. Next day, cells were incubated with culture medium in the presence or absence of $5 \mu \mathrm{M}$ PLX4032 for 3 weeks, with refreshment of medium twice a week. The cells were then fixed with $10 \%$ cold methanol for $5 \mathrm{~min}$ and stained with $0.005 \%(\mathrm{~m} / \mathrm{v})$ crystal violet for $30 \mathrm{~min}$ at room temperature. After extensive wash, the colonies were photographed.

ChIP assay. Mel-CV and Mel-CVR with or without CREB stably knockdown were crosslinked with $1 \%$ formaldehyde for $10 \mathrm{~min}$ at room temperature. ChIP assay was performed according to the manufacturer's instructions by using antiCREB and the ChIP assay kit (Millipore, Merck KGaA, Darmstadt, Germany). Antirabbit IgG were used as controls. The bound DNA fragments were eluted and amplified by PCR using the following primer pair: $5^{\prime}$-GCAAC CAAACTTCTCTGTCC- $3^{\prime}$ and $5^{\prime}$-TCCTGCCTCAGCCTCCCAA- $3^{\prime}$. PCR products were separated on $2 \%$ agarose gel by gel electrophoresis.

Xenograft mouse model. Mel-CV and Mel-CVR cells with and without stable knockdown of CREB or AEBP1 (about $1 \times 10^{7}$ ) were injected subcutaneously into the right flank of nude mice (Shanghai SLAC Laboratory, Shanghai, China). Fifteen days after injection, palpable tumors were present in all mice. The mice bearing tumor of similar size $\left(\right.$ about $50 \mathrm{~mm}^{3}$ ) from each group $(n=6)$ were treated with PLX4032 $(50 \mathrm{mg} / \mathrm{kg})$ through i.p. injection. Twenty-five days after PLX4032 treatment, mice were killed and tumors were excised and weighed. Studies on animals were conducted with approval from the Animal Research Ethics Committee of University of Science and Technology of China.

Patient tissues. Ten tumor samples were collected from five metastatic melanoma patients treated with a BRAF inhibitor as part of the phase I $(n=2)$, and phase II-brain metastases $(n=2)$ clinical trials of dabrafenib, ${ }^{7,41}$ or the expanded access program for vemurafenib $(n=1)$. Patients eligible for these studies had unresectable AJCC stage III or stage IV BRAF mutant melanoma and were treated with either dabrafenib (150 mg, twice daily) or vemurafenib $(960 \mathrm{mg}$ twice daily). Biopsied tumor specimens were collected from consenting patients before BRAF inhibitor treatment and at disease progression, as part of the Treat Excise Analyze for Melanoma Study at the Melanoma Institute Australia, Sydney, Australia, as approved by the Royal Prince Alfred Hospital Research Ethics Committee Protocol No X10-0305 \& HREC/10/RPAH.

\section{Conflict of Interest}

The authors declare no conflict of interest.
Acknowledgements. We are grateful to Dr. Peter Heresy for establishment of the primary melanoma cell lines from paired melanoma samples pre- and posttreatment with vemurafenib; Professor Richard Kefford, Jessica Hyman, James Wilmott, Helen Rizos and the MIA surgeons, specifically Julie Howle, Kerwin Shannon, Robyn Saw, John Thompson, Andrew Spillane and Jonathan Stretch for their assistance in procuring melanoma specimens; and Dr. Hyo-Sung Ro for kindly providing us with pCDNA-AEBP1 plasmid. This work was supported by grants from the Ministry of Science and Technology of China (2010CB912804 and 2011CB966302); National Natural Science Foundation of China 31030046 and the Fundamental Research Funds for Central Universities (WK2060190018). The project was also partly supported by Open Research Fund of State Key Laboratory of Cellular Stress Biology, Xiamen University to Qiao Wu.

1. Davies H, Bignell GR, Cox C, Stephens P, Edkins S, Clegg $S$ et al. Mutations of the BRAF gene in human cancer. Nature 2002; 417: 949-954.

2. Wan PT, Garnett MJ, Roe SM, Lee S, Niculescu-Duvaz D, Good VM et al. Mechanism of activation of the RAF-ERK signaling pathway by oncogenic mutations of B-RAF. Cell 2004; 116: 855-867.

3. Dhomen N, Marais R. New insight into BRAF mutations in cancer. Curr Opin Genet Dev 2007; 17: 31-39.

4. Tsai J, Lee JT, Wang W, Zhang J, Cho H, Mamo S et al. Discovery of a selective inhibitor of oncogenic B-Raf kinase with potent antimelanoma activity. Proc Natl Acad Sci USA 2008; 105: 3041-3046.

5. Chapman PB, Hauschild A, Robert C, Haanen JB, Ascierto P, Larkin J et al. Improved survival with vemurafenib in melanoma with BRAF V600E mutation. N Engl J Med 2011; 364: 2507-2516.

6. Flaherty KT, Puzanov I, Kim KB, Ribas A, McArthur GA, Sosman JA et al. Inhibition of mutated, activated BRAF in metastatic melanoma. N Engl J Med 2010; 363: 809-819.

7. Falchook GS, Long GV, Kurzrock R, Kim KB, Arkenau TH, Brown MP et al. Dabrafenib in patients with melanoma, untreated brain metastases, and other solid tumours: a phase 1 dose-escalation trial. Lancet 2012; 379: 1893-1901.

8. Hauschild A, Grob JJ, Demidov LV, Jouary T, Gutzmer R, Millward M et al. Dabrafenib in BRAF-mutated metastatic melanoma: a multicentre, open-label, phase 3 randomised controlled trial. Lancet 2012; 380: 358-365.

9. Johannessen CM, Boehm JS, Kim SY, Thomas SR, Wardwell L, Johnson LA et al. COT drives resistance to RAF inhibition through MAP kinase pathway reactivation. Nature 2010; 468: 968-972.

10. Montagut C, Sharma SV, Shioda T, McDermott U, UIman M, UIkus LE et al. Elevated CRAF as a potential mechanism of acquired resistance to BRAF inhibition in melanoma. Cancer Res 2008; 68: 4853-4861.

11. Nazarian R, Shi H, Wang Q, Kong $\mathrm{X}$, Koya RC, Lee $\mathrm{H}$ et al. Melanomas acquire resistance to B-RAF(V600E) inhibition by RTK or N-RAS upregulation. Nature 2010; 468: 973-977.

12. Shi H, Moriceau G, Kong X, Lee MK, Lee H, Koya RC et al. Melanoma whole-exome sequencing identifies (V600E)B-RAF amplification-mediated acquired B-RAF inhibitor resistance. Nat Commun 2012; 3: 724.

13. Villanueva J, Vultur A, Lee JT, Somasundaram R, Fukunaga-Kalabis M, Cipolla AK et al. Acquired resistance to BRAF inhibitors mediated by a RAF kinase switch in melanoma can be overcome by cotargeting MEK and IGF-1R/PI3K. Cancer Cell 2010; 18: 683-695.

14. Yadav V, Zhang X, Liu J, Estrem S, Li S, Gong XQ et al. Reactivation of Mitogen-activated Protein Kinase (MAPK) Pathway by FGF Receptor 3 (FGFR3)/Ras Mediates Resistance to Vemurafenib in Human B-RAF V600E Mutant Melanoma. J Biol Chem 2012; 287: 28087-28098.

15. Poulikakos PI, Persaud Y, Janakiraman M, Kong X, Ng C, Moriceau G et al. RAF inhibitor resistance is mediated by dimerization of aberrantly spliced BRAF(V600E). Nature 2011; 480: 387-390.

16. Basile KJ, Abel EV, Aplin AE. Adaptive upregulation of FOXD3 and resistance to PLX4032/4720-induced cell death in mutant B-RAF melanoma cells. Oncogene 2012; 31: 2471-2479.

17. Prahallad A, Sun C, Huang S, Di Nicolantonio F, Salazar R, Zecchin D et al. Unresponsiveness of colon cancer to BRAF(V600E) inhibition through feedback activation of EGFR. Nature 2012; 483: 100-103.

18. Sandelin A, Alkema W, Engstrom P, Wasserman WW, Lenhard B. JASPAR: an open-access database for eukaryotic transcription factor binding profiles. Nucleic Acids Res 2004; 32(Database issue): D91-D94.

19. Du K, Montminy M. CREB is a regulatory target for the protein kinase Akt/PKB. J Biol Chem 1998; 273: 32377-32379.

20. Mei Y, Xie C, Xie W, Tian X, Li M, Wu M. Noxa/Mcl-1 balance regulates susceptibility of cells to camptothecin-induced apoptosis. Neoplasia 2007; 9: 871-881.

21. Majdalawieh A, Zhang L, Ro HS. Adipocyte enhancer-binding protein-1 promotes macrophage inflammatory responsiveness by up-regulating NF-kappaB via IkappaBalpha negative regulation. Mol Biol Cell 2007; 18: 930-942. 
22. Gray-Schopfer VC, Karasarides M, Hayward R, Marais R. Tumor necrosis factor-alpha blocks apoptosis in melanoma cells when BRAF signaling is inhibited. Cancer Res 2007; 67: 122-129.

23. Lai $F$, Jiang $C C$, Farrelly ML, Zhang XD, Hersey P. Evidence for upregulation of Bim and the splicing factor SRp55 in melanoma cells from patients treated with selective BRAF inhibitors. Melanoma Res 2012; 22: 244-251.

24. He GP, Muise A, Li AW, Ro HS. A eukaryotic transcriptional repressor with carboxypeptidase activity. Nature 1995; 378: 92-96.

25. Muise AM, Ro HS. Enzymic characterization of a novel member of the regulatory B-like carboxypeptidase with transcriptional repression function: stimulation of enzymic activity by its target DNA. Biochem J 1999; 343(Pt 2): 341-345.

26. Kim SW, Muise AM, Lyons PJ, Ro HS. Regulation of adipogenesis by a transcriptional repressor that modulates MAPK activation. J Biol Chem 2001; 276: 10199-10206.

27. Majdalawieh A, Zhang L, Fuki IV, Rader DJ, Ro HS. Adipocyte enhancer-binding protein 1 is a potential novel atherogenic factor involved in macrophage cholesterol homeostasis and inflammation. Proc Natl Acad Sci USA 2006; 103: 2346-2351.

28. Park JG, Muise A, He GP, Kim SW, Ro HS. Transcriptional regulation by the gamma5 subunit of a heterotrimeric $G$ protein during adipogenesis. EMBO J 1999; 18: 4004-4012.

29. Ozgen N, Obreztchikova M, Guo J, Elouardighi H, Dorn GW 2nd, Wilson BA et al. Protein kinase $D$ links $\mathrm{Gq}$-coupled receptors to cAMP response element-binding protein (CREB)Ser133 phosphorylation in the heart. J Biol Chem 2008; 283: 17009-17019.

30. Xing J, Kornhauser JM, Xia Z, Thiele EA, Greenberg ME. Nerve growth facto activates extracellular signal-regulated kinase and p38 mitogen-activated protein kinase pathways to stimulate CREB serine 133 phosphorylation. Mol Cell Biol 1998; 18 : 1946-1955.

31. Wilson TR, Fridlyand J, Yan Y, Penuel E, Burton L, Chan E et al. Widespread potential for growth-factor-driven resistance to anticancer kinase inhibitors. Nature 2012; 487: 505-509.

32. Straussman R, Morikawa T, Shee K, Barzily-Rokni M, Qian ZR, Du J et al. Tumour microenvironment elicits innate resistance to RAF inhibitors through HGF secretion. Nature 2012; 487: 500-504.
33. Jiang CC, Lai F, Thorne RF, Yang F, Liu H, Hersey $P$ et al. MEK-independent survival of B-RAFV600E melanoma cells selected for resistance to apoptosis induced by the RAF inhibitor PLX4720. Clin Cancer Res 2011; 17: 721-730.

34. Shao Y, Aplin AE. Akt3-mediated resistance to apoptosis in B-RAF-targeted melanoma cells. Cancer Res 2010; 70: 6670-6681.

35. Ro HS, Zhang L, Majdalawieh A, Kim SW, Wu X, Lyons PJ et al. Adipocyte enhancerbinding protein 1 modulates adiposity and energy homeostasis. Obesity (Silver Spring) 2007; 15: 288-302.

36. Ozes ON, Mayo LD, Gustin JA, Pfeffer SR, Pfeffer LM, Donner DB. NF-kappaB activation by tumour necrosis factor requires the Akt serine-threonine kinase. Nature 1999; 401: 82-85.

37. Koul D, Yao Y, Abbruzzese JL, Yung WK, Reddy SA. Tumor suppressor MMAC/PTEN inhibits cytokine-induced NFkappaB activation without interfering with the IkappaB degradation pathway. J Biol Chem 2001; 276: 11402-11408.

38. Ueda Y, Richmond A. NF-kappaB activation in melanoma. Pigment Cell Res 2006; 19 : 112-124.

39. Yang J, Amiri KI, Burke JR, Schmid JA, Richmond A. BMS-345541 targets inhibitor of kappaB kinase and induces apoptosis in melanoma: involvement of nuclear factor kappaB and mitochondria pathways. Clin Cancer Res 2006; 12(3 Pt 1): 950-960.

40. Zhang XD, Zhang XY, Gray CP, Nguyen T, Hersey P. Tumor necrosis factor-related apoptosis-inducing ligand-induced apoptosis of human melanoma is regulated by smac/DIABLO release from mitochondria. Cancer Res 2001; 61: 7339-7348.

41. Long GV, Trefzer U, Davies MA, Kefford RF, Ascierto PA, Chapman PB et al. Dabrafenib in patients with Val600Glu or Val600Lys BRAF-mutant melanoma metastatic to the brain (BREAK-MB): a multicentre, open-label, phase 2 trial. Lancet Oncol 2012; 13: 1087-1095.

cc) (5) $\Theta$ Cell Death and Disease is an open-access journal BY ${ }_{\text {NC }}$ published by Nature Publishing Group. This work is licensed under a Creative Commons Attribution-NonCommercialNoDerivs 3.0 Unported License. To view a copy of this license, visit http://creativecommons.org/licenses/by-nc-nd/3.0/

Supplementary Information accompanies this paper on Cell Death and Disease website (http://www.nature.com/cddis) 